RESEARCH ARTICLE

\title{
In vivo plasmid DNA electroporation generates exceptionally high levels of epitope-specific CD8+ $T$-cell responses
}

\author{
W Paster ${ }^{1}$, M Zehetner ${ }^{1}$, M Kalat ${ }^{1}$, S Schüller ${ }^{2}$ and T Schweighoffer ${ }^{1}$ \\ ${ }^{1}$ Department of NBE Discovery, Boehringer Ingelheim Austria, Vienna, Austria; and ${ }^{2}$ Gene Therapy Group, Boehringer Ingelheim \\ Austria, Vienna, Austria
}

\begin{abstract}
Based on observations that DBA/2 mice develop a highly specific response towards an HLA-Cw3-derived epitope, consisting entirely of CD8+CD62L-V $\beta 10+$ cells, we have established an in vivo mouse model for screening a variety of immunization approaches. Responder cells were readily detectable in small samples of the peripheral blood using three-color FACS analysis. This permitted multiple, sequential determination of CD8+ T-cell responses in living animals at a very high degree of precision. In vivo electroporation delivery of expression construct plasmids, outclassed the other approaches tested. Dominant, specific responses were induced already upon a single administration. Both the peak
\end{abstract}

and the longevity of the response resembled those that are generated by the most active viral infections. The induced CTLs rejected epitope-bearing tumor cells in vivo and released interferon- $\gamma$ upon stimulation with the correct MHC::peptide combination in vitro. The potent in vivo response was not influenced by known modulators of the innate immune system, such as CpG DNA and LPS content. In vivo electroporation thus deserves consideration in the future in antitumor and antiviral immunization approaches, where $C D 8+T$ cells play a predominant role.

Gene Therapy (2003) 10, 717-724. doi:10.1038/sj.gt.3301942

Keywords: memory; MHC; CD8+ T lymphocytes; electroporation; mouse model

\section{Introduction}

Responses provided by CD8+ T cells play a pivotal role in host defense by recognizing viral or tumor-derived antigenic epitopes as well as epitopes of bacterial and parasitic origin. Only recently it had been defined to what enormous extent CD8+ T-cell expansion takes place during natural viral infections. ${ }^{1}$ These peak responses routinely leave a proportionally sized memory population behind. ${ }^{2}$ A new generation of successful vaccines should achieve high frequencies of specific cells, but should be safe, simple, stable, and affordable at the same time. Although there have been substantial advances in the field, an optimal solution has not been achieved yet.

In an effort to screen and to compare a broad variety of innovative molecular and cellular vaccine approaches, we needed a noninfectious in vivo mouse model, which at the same time also enabled precise determination of CD8+ T-cell responses at least at a medium throughput rate. We have successfully established a model that meets these criteria by adapting a previously described unique observation. Janet Maryanski and co-workers described that live P815 mastocytoma cells, transfected with the human HLA Cw3 gene, reproducibly induced large-scale

Correspondence: W Paster, Current address: Vienna International Research Cooperation Centers, Brunnerstraße 59, Vienna A-1235 Wien, Vienna, Austria.E-mail: wolfgang.paster@univie.ac.at

Received 2 September 2002; accepted 22 October 2002 expansion of CD8+ T cells in DBA/2 mice. ${ }^{3}$ Responder cells were specific for the Cw3 protein-derived Cw3170179 RYLKNGKETL (RYLK) epitope, restricted by the MHC class I Kd element. High levels of specific CD8+ T cells could be found both in lymphoid organs and in peripheral blood. Moreover, only an extremely limited repertoire of $\mathrm{T}$ cells formed the response, all of which exclusively utilized the TCR V $\beta 10$ segment. ${ }^{4,5}$ CD62L was absent on these cells. ${ }^{6}$ Thus, the unique phenotype of CD8+CD62L-V $\beta 10+$ enabled their detection with high precision even from small aliquots of peripheral blood. ${ }^{7}$

We have screened numerous immunization protocols on the basis of the Cw3 model. These included, among others, cellular vaccines, virus particles, native and modified peptides, and nucleic acid-based vaccines. One approach where we were able to recapitulate both the magnitude and the longevity of a high-efficiency CTL response was by administration of a plasmid construct using optimized in vivo electroporation (EP). Electrical pulses have been reported to enhance gene expression from plasmid DNA in laboratory animals, and to produce elevated antibody titers in mice against $\mathrm{HBsAg}$ and HIV gag. 8,9 Zucchelli et al also found enhanced Band T-cell responses against hepatitis $C$ virus E2 using a similar technique. ${ }^{10}$

In our hands, in vivo EP demonstrated key characteristics that have not been previously achieved by synthetic molecular vaccines. Clonal burst size and longevity of memory cells rivaled the most vigorous CD8+ T-cell expansions that could be induced by live 
immunogens. Immunized animals rejected a tumor challenge and their spleen cells recognized appropriate MHC-target peptide combinations. LPS and CpG content did not considerably influence the potent Cw3 response. Most importantly, these features were available already after a single application. Provided that these results are also pertinent in other systems, this very effective yet uncomplicated in vivo EP protocol undoubtedly deserves to be tested for human applications.

\section{Results}

\section{In vivo EP of plasmid DNA induces very high peak responses and leaves a functionally active, long-lived population behind}

The unique properties of the Cw3 model system enabled us to measure specific $\mathrm{T}$-cell responses from small samples of peripheral blood. Thus, mice had not to be killed at each measurement and serial data could be obtained from single animals over a long period. High-affinity staining techniques with sensitive FACS readout enabled fast and precise determination of the response.

Based on our model antigen system, numerous vaccination approaches were tested. Table 1 gives an overview of the different immunogens used and their effectiveness in inducing CD8+ T-cell expansion. Cellular vaccines, that is, stable transfectants of MHC-matched P815 cells expressing HLA Cw3 or parts thereof, were by far the most successful vaccines. Among these, best results were consistently achieved by injection of P815Cw3 cells, whereas P815 cells alone or P815 cells expressing the dominant RYLK-epitope fused to EGFP induced no or only marginal expansion of specific CD8+ $\mathrm{T}$ cells. Irradiated P815 transfectants were also only barely immunogenic. Peptide vaccines generally were inefficient even when used together with adjuvants, of which at least polyarginine showed some effect, as did lipid modification with palmitoyl chains. ${ }^{11-13}$ Adding negatively charged residues in order to neutralize the basic residues of the epitope proved to be as ineffective as extending the peptide both C- and N-terminally. Another approach made use of a baculovirus expression system, which was one of the more successful immunization regimens. ${ }^{14,15}$

In contrast to the above methods that mostly induced only small numbers of specific CD8+ T cells, outstanding results were consistently achieved by EP-enhanced delivery of various plasmid constructs carrying the full-length human HLA-Cw*0303 coding sequence. Specific CD8+ T cells made up $15-25 \%$ of the total peripheral CD8+ T pool when $50 \mu \mathrm{g}$ plasmid DNA per animal was injected. Individual high-responder mice were often above these levels. As a prominent example, mouse C165-5 had $0.3 \%$ specific CD8+ cells before vaccination (Figure 1a). The same mouse, after a single EP application, displayed $43.3 \%$ specific CD8+CD62L$\mathrm{V} \beta 10+\mathrm{T}$ cells (Figure 1b).

From time-course experiments taken in the same animals with serial sampling, we determined that the response peaked 12-13 days after priming. Thus, the kinetic of the expansion appeared to be somewhat delayed in comparison to that seen with live immunogens. ${ }^{1,16}$ Specific CD8+ T cells declined then at similar rate in each mouse in a group of four (Figure 2a; note the logarithmic scaling of the $y$-axis depicting the percentspecific CD8+ T cells). In control mice, measurable hGH levels in plasma, but no expansion of CD8+CD62L$\mathrm{V} \beta 10+\mathrm{T}$ cells could be obtained when a plasmid encoding hGH instead of Cw3 was injected (Figure 2b), or when the Cw3 plasmid was delivered without EP (Figure 2c).

Besides DBA/2 mice, in vivo EP has been tested on B6D2F1 mice. This strain not only carries the $\mathrm{H}-2 \mathrm{~b}$ alleles in addition to $\mathrm{H}-2 \mathrm{~d}$, but also has a different $\mathrm{T}$-cell receptor repertoire. In vivo EP induced high numbers of specific CD8+ T cells with a similar kinetic also in these mice (Figure 2d).

We have followed up specific T-cell responses for more than 1 year after a single EP administration by repeatedly testing a cohort of mice. As expected from the very high peak response, the number of specific CD8+ $\mathrm{T}$ cells detectable in the circulation reapproached the limit of

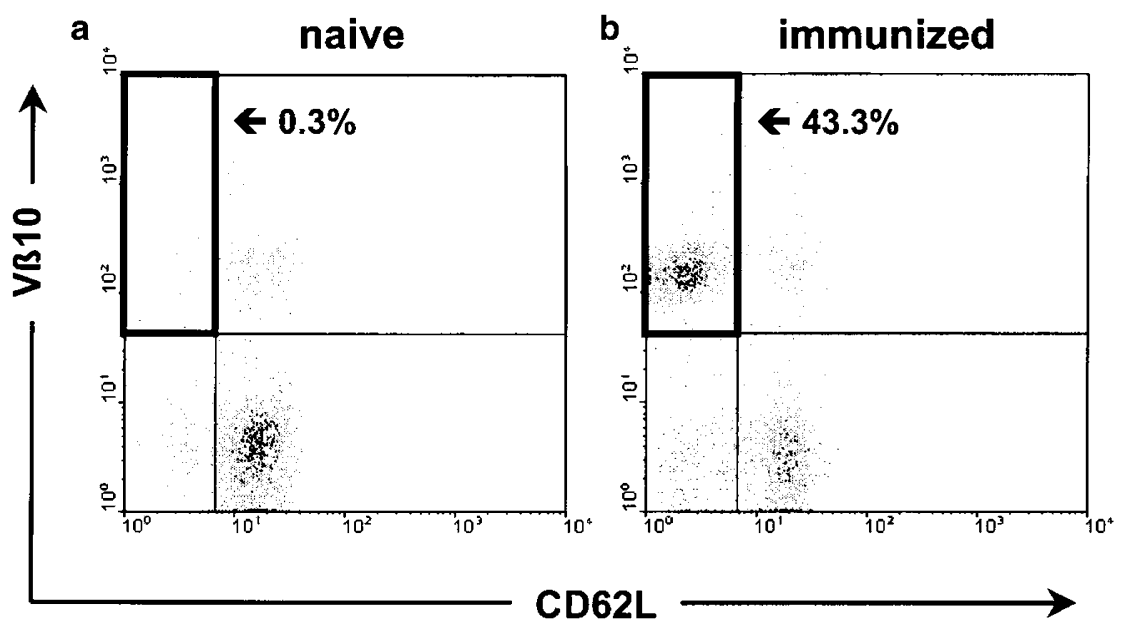

Figure 1 Plasmid DNA delivered by in vivo EP triggers expansion of antigen epitope-specific CD8+T cells in DBA/2 mice. Peripheral blood lymphocytes were stained for flow cytometric analysis with anti-CD8, anti-CD62L, and anti-TCR-VB10 antibodies. The data shown are gated on live CD8+T cells, and are from the same animal 1 day before vaccination (a) and 12 days after vaccination (b). 

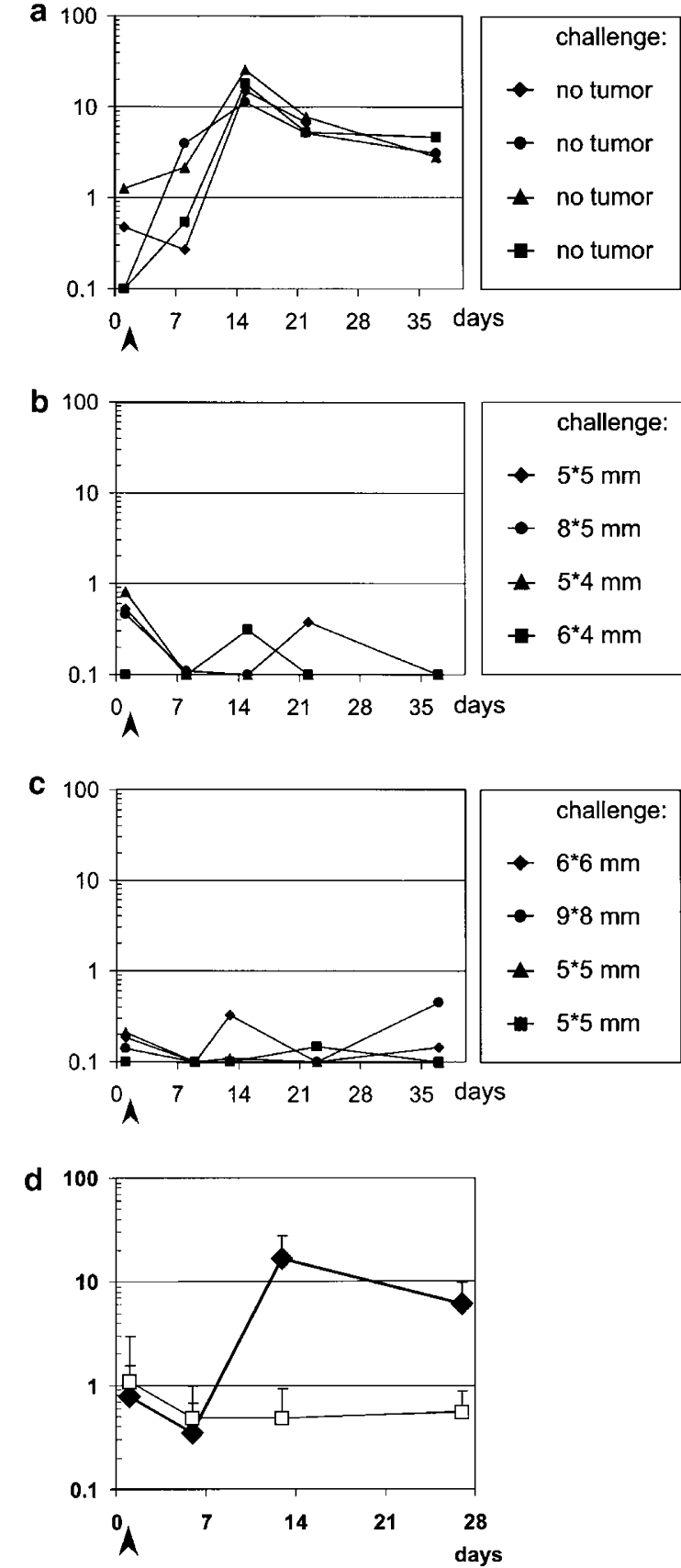

Figure 2 Specific CD8+ cell responses in the peripheral blood. Groups of four mice are shown that received on day 1 (arrowhead) $50 \mu \mathrm{g}$ Cw3 plasmid plus EP (a); $50 \mu \mathrm{g}$ hGH plasmid plus EP (b); and $50 \mu \mathrm{g}$ Cw3 plasmid without EP (c). Each symbol corresponds to an individual animal. Mice were challenged 43 days after immunization with $10^{6}$ P815\#123 cells subcutaneously. Tumor sizes measured on day 55 are shown adjacent to panels (a), (b), and (c); symbols correspond to individual mice. (d) Groups of four F1 hybrid B6D2F1 animals carrying both $\mathrm{H}-2 \mathrm{~b}$ and $\mathrm{H}-2 \mathrm{~d}$ haplotypes were left untreated $(\square)$ or received a single EP (arrowhead) with $50 \mu \mathrm{g}$ Cw3 plasmid DNA ( ). Average+s.d. of the group shown. Note the logarithmic scale of response data.

detection (LOD, for calculation see The Material and methods section) only after more than 200 days (Figure 3). The best mathematical equation describing these long-term data fits a three-compartment model, and suggests that specific CD8+ T cells, which survive the phase of rapid decline (apoptosis), are eliminated from the circulation only very slowly if at all.
$C D 8+T$ cells induced by in vivo EP release interferon- $\gamma$ (INF- $\gamma$ ) after stimulation and protect against tumor challenge

Functional competence of CD8+ T cells obtained after a single vaccination was tested in two different assays. First, a tumor challenge experiment was performed with P815\#123 cells. These cells carried the minimal H-2Kd restricted Cw3 epitope fused to the C-terminus of EGFP, and are thus a suitable target for immune cells, but are themselves only weakly immunogenic (see also Table 1). The same mice that were immunized once with $50 \mu \mathrm{g}$ of plasmid pVAX.Cw3 with or without EP or electroporated with $50 \mu \mathrm{g}$ pVAX.hGH plasmid, received a subcutaneous inoculum of $10^{6}$ live cells ( $\sim 100$-fold tumorigenic dose) on day 43. Tumor growth correlated inversely with the development of a dominant CTL response (Figure 2a-c; right-side panels).

Second, CTLs obtained from vaccinated animals were tested for INF- $\gamma$ production. As target, the H-2Kdpositive C26 carcinoma line was used (Figure 4a). C26 cells alone did not produce IFN- $\gamma$, nor did CTLs in the presence of untransfected C26 cells. CTLs, however, produced high amounts of IFN- $\gamma$ when cocultured with cells that displayed the correct MHC::peptide combination: both P815-Cw3 cells or C26 cells transiently transfected with pVAX.Cw3 stimulated IFN- $\gamma$ release. C26 cells transfected with control plasmids, such as pVAX.H2Kb or pVAX.H2Kd did not stimulate the CTLs.

COS7 transfectants were also tested as targets (Figure $4 \mathrm{~b})$. These cells are of green monkey origin, and carry HLA homologues that contain the RYLK motif, but they lack the appropriate $\mathrm{H}-2 \mathrm{Kd}$ restriction element. Thus, only when transfected with pVAX.H2Kd were these cells recognized by the CTLs.

\section{CpG or LPS content do not significantly influence efficiency of in vivo EP}

Bacterial DNA, and more specifically its $\mathrm{CpG}$ content has been widely shown to promote the initiation of the immune reposes via TLR-9 receptors present on a subset of antigen-presenting cells. ${ }^{17}$ To test whether the CpG content of the plasmid backbone has any consequence for the vaccination efficiency in our model antigen system, we prepared four different vectors. As CPG content is contributed dominantly by the coding sequence of the $\beta$-lactamase gene whose product is conferring ampicillin resistance, we have compared plasmids with ampicillin and kanamycin resistance. ${ }^{18}$ To account for differences in the promoter region, the generic CMV promoter was also replaced by the equally effective human EF $\alpha$ promoter. ${ }^{19}$ Data shown in Figure 5a indicate that increased CpG content of the ampicillinresistant vectors (open symbols) actually deteriorated the response instead of further enhancing it. Plasmids with the kanamycin resistance marker (closed symbols) performed nearly equally well with both the CMV and the $\mathrm{EF} \alpha$ promoter.

CpG-containing oligonucleotides that were shown previously to successfully enhance in vivo immune responses in mice were admixed to the standard pVAX.Cw3 vector, and coelectroporated with it. ${ }^{20}$ Again, no difference was found between the stimulatory CpG oligo 1826 and the control oligonucleotide 1982 


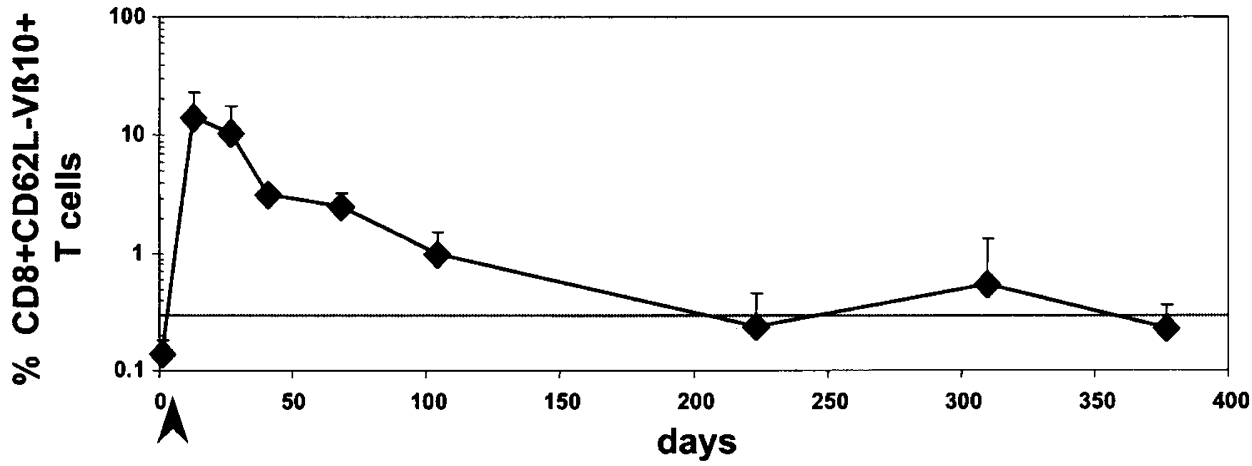

Figure 3 A single application of $50 \mu \mathrm{g}$ plasmid on day 1 induces responses that are above detection limit even after 1 year. Peripheral blood of mice that received a single vaccination (arrowhead) with $50 \mu \mathrm{g}$ Cw3 plasmid plus EP on day 1 were repeatedly tested by FACS staining. Average+s.d. of the group of four mice is shown. The dotted line indicates the LOD value, calculated from $>500$ nave, untreated or other control animals and is $0.3 \pm 0.1 \%$.

Table 1 Comparison of different Cw3-based immunogens

\begin{tabular}{|c|c|c|c|}
\hline Immunogen & & Animals with peak above $L O D$ & Highest detected response \% specific cells \\
\hline \multirow{4}{*}{ Cellular, live } & P815, (2E6) & $0 / 4$ & - \\
\hline & P815-Cw3 full length (2E7 cells $\left.{ }^{a}\right)$ & $10 / 10$ & 37.9 \\
\hline & P815\#123 epitope (1E6 cells $\left.{ }^{a}\right)$ & $2 / 15$ & 3.1 \\
\hline & & & - \\
\hline \multirow{2}{*}{ Cellular, irradiated } & P815-Cw3 full length (1E7 cells) & $3 / 3$ & 3.6 \\
\hline & P815\#123 full length (1E6 cells) & $0 / 3$ & - \\
\hline \multirow[t]{6}{*}{ Peptides } & RYLKNGKETL & $0 / 11$ & - \\
\hline & RYLKNGKETL+polylysine & $0 / 8$ & - \\
\hline & RYLKNGKETL+polyarginin & $2 / 3$ & 0.6 \\
\hline & palmitoyl - GRYLKNGKETL & $1 / 7$ & 0.8 \\
\hline & RYLKNGKETLEE & $0 / 10$ & - \\
\hline & LRRYLKNGKETLQGAE & $0 / 2$ & - \\
\hline \multirow[t]{2}{*}{ Baculovirus } & vBACw3 & $0 / 3$ & - \\
\hline & vBACMV3 & $1 / 3$ & 2.7 \\
\hline
\end{tabular}

aThese were the highest cell numbers that were possible to apply; otherwise, rapid tumor outgrowth led necessarily to premature termination of the experiments.

(Figure 5b) (numbers are the original designations of the oligonucleotides from Davis et $a l^{20}$ ).

Finally, the effects of E. coli-derived LPS were tested, which on the one hand is a frequent contaminant of plasmid preparations and decreases transfection efficiency in vitro, and may drive cells in apoptosis through TLR-2, but on the other hand also stimulates the innate immune system through TLR-4. ${ }^{21-23}$ Different concentrations of LPS were coadministered with the standard pVAX.Cw3. No dose-response relations could be found between the amount of LPS and the percent of specific CD8+ $\mathrm{T}$ cells generated (Figure 5c). When $50 \mu \mathrm{g}$ of plasmid was electrovaccinated, the CD8+ responses remained essentially stable; with $10 \mu \mathrm{g}$ plasmid per mouse, the response declined only when higher amounts of LPS were applied.

\section{Discussion}

With this work we had two major aims: to set up an in vivo mouse model system that enables rapid yet accurate comparison of various immunization methods, and also to design immunization protocols that yield exceptionally high and long-lasting CD8+ T-cell responses.
For our needs, the Cw3-based immunization model proved to be a valuable and practical tool. We could take advantage of the facts that (a) DBA/2 mice are highly reactive towards the RYLK epitope and (b) that responder cells bear the exclusive CD8+CD62L-V $\beta 10+$ phenotype, readily detectable by three-color FACS analysis. However, several refinements had to be added before this unique constellation could be used as a vaccine model. First, we had to restrict ourselves to small (up to $200 \mu \mathrm{l})$ amounts of peripheral blood as test material, as we wanted to monitor individual animals repeatedly for extended periods of time. It is very important that this type of readout did not require mice with transgenic modification or any further ex vivo manipulations. Second, only live transfected cells were previously shown to function as effective immunogen. The refined detection method was perfectly adequate for such a high degree in vivo CD8+ T-cell expansion. For molecular vaccines, however, it was completely unclear whether we could induce responses that exceed the LOD of $0.3 \pm 0.1 \%$. Published immunization protocols only rarely reached this critical value. Several approaches that we have tested, including peptides of various length containing the RYLK epitope, palmitoylated peptides and 


\section{$\mathrm{pg} / \mathrm{ml} \mathrm{IFN}-\gamma$}
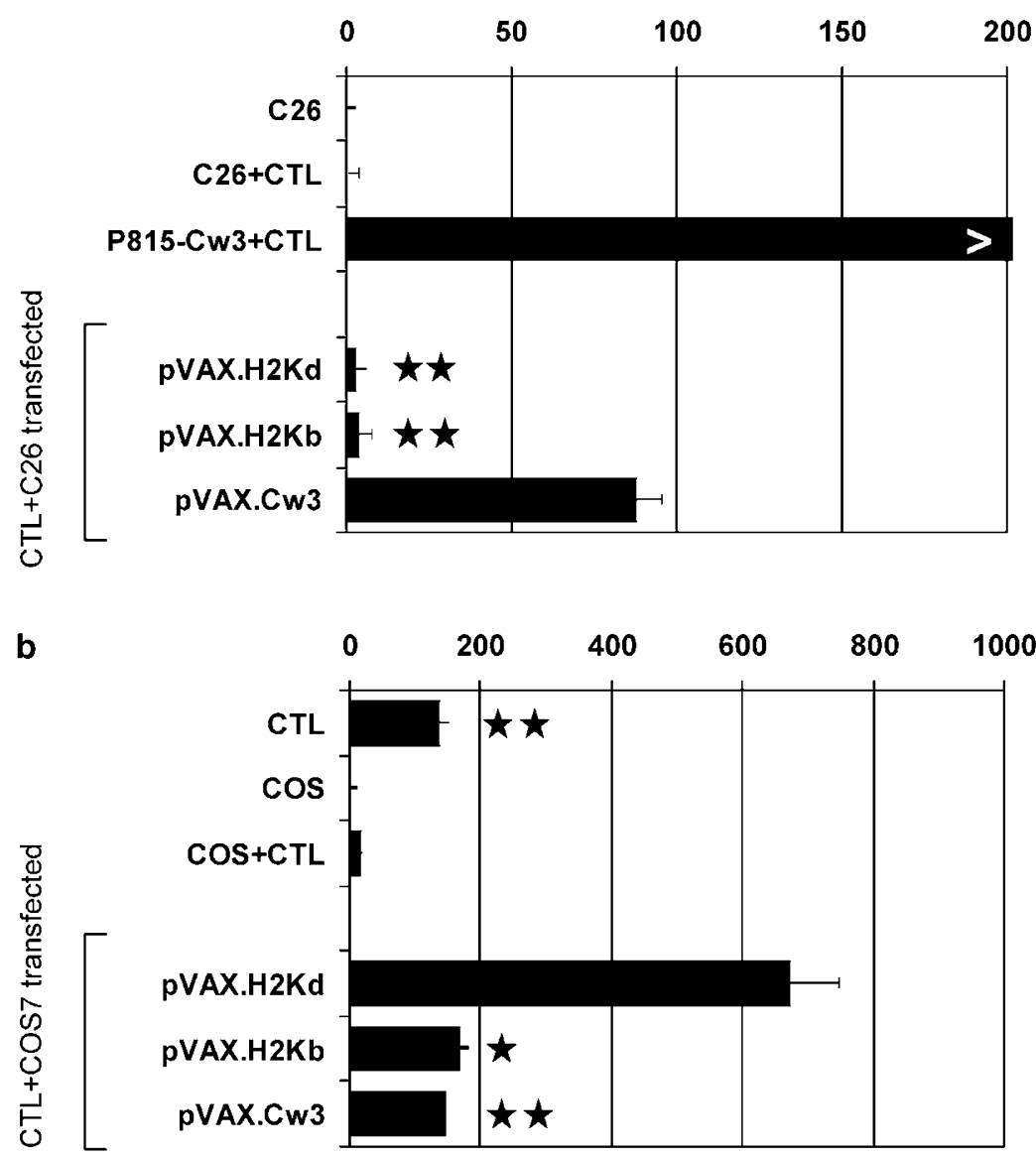

Figure 4 CD8+ Tcells elicited by in vivo EP release IFN- $\gamma$ upon stimulation with the correct MHC::epitope complex. CD8+ Tcells isolated from the spleen of electrovaccinated animals were cocultured with transfected C26 cells $(\mathrm{H}-2 \mathrm{Kd}+$, panel a) or COS7 cells (green monkey origin, carries HLA homologues which contain the RYLK motif; panel b). After, 4 days of cocultivation, the IFN- $\gamma$ content of the supernatants was measured. Statistical comparisons between several data sets and the relevant transfectants ( $p V A X . C w 3$ in panel a, $p V A X . H 2 K d$ in panel $b$ ) were done using Student's $t$-test. Two asterisks denote a statistical significance of $P<0.005$, one asterisk stands for $P<0.01$. Cultures were done in triplicates and data shown are from one representative experiment out of three repetitions.

peptide-carrier complexes, in fact, rarely produced a response in the detectable range. ${ }^{11-13,24}$

We have been able to induce exceptionally potent CD8+ T-cell responses by using a purely molecular plasmid DNA vaccine administered by the novel technique of in vivo electroperation (EP). Here the administration of a buffered plasmid DNA solution, followed by EP at the site of the injection, results in massive immune responses. As plasmids are nonreplicating in mammalian cells, exact control of antigen doses was possible, which is not the case with live cellular or viral immunogens. Following a single dose of our model antigen HLA-Cw3, immunity was long lived, as shown by long-term (more than 1 year) studies of individual mice, and protective against tumor challenges. In vivo EP was shown to dramatically enhance responses when compared to injection of naked DNA alone. Specificity and functional competence of responder CD8+ T cells raised after a single immunization was also proven in vitro.

So far, the general impression was that synthetic molecular vaccines were far less effective compared to live immunogens, such as viruses, persistent bacteria, or even live cells. The most promising DNA prime - live vaccinia virus boost protocols or DNA/cytokine combinations still needed multiple applications to reach a 1 $4 \%$ range of CD8+ T-cell expansion. ${ }^{25,26}$ What are thus the critical factors that facilitate the observed burst of CD8+ T cells upon EP in the Cw3 system?

One important factor, as previously shown, is clearly the high level of $\mathrm{Ag}$ expression at the site of application. ${ }^{27,28}$ Besides the local expression by myocytes or keratinocytes, there is growing evidence that dendritic cells (DCs) as rapidly migrating professional antigenpresenting cells play a major role in plasmid DNA vaccination. DCs are directly transfected or may take up $\mathrm{Ag}$ that is secreted by factory cells or released by apoptotic cells. ${ }^{29}$ One may assume that these processes are even more effective after EP. Also, physical consequences of EP together with the unavoidable death of some transfected cells may supply danger signals, efficiently activating DCs.

Providing additional 'danger' as done by admixing CPG oligos or LPS did not appear to enhance EP in our system. Only when decreasing the DNA concentration, and therefore the response magnitude, some negative 

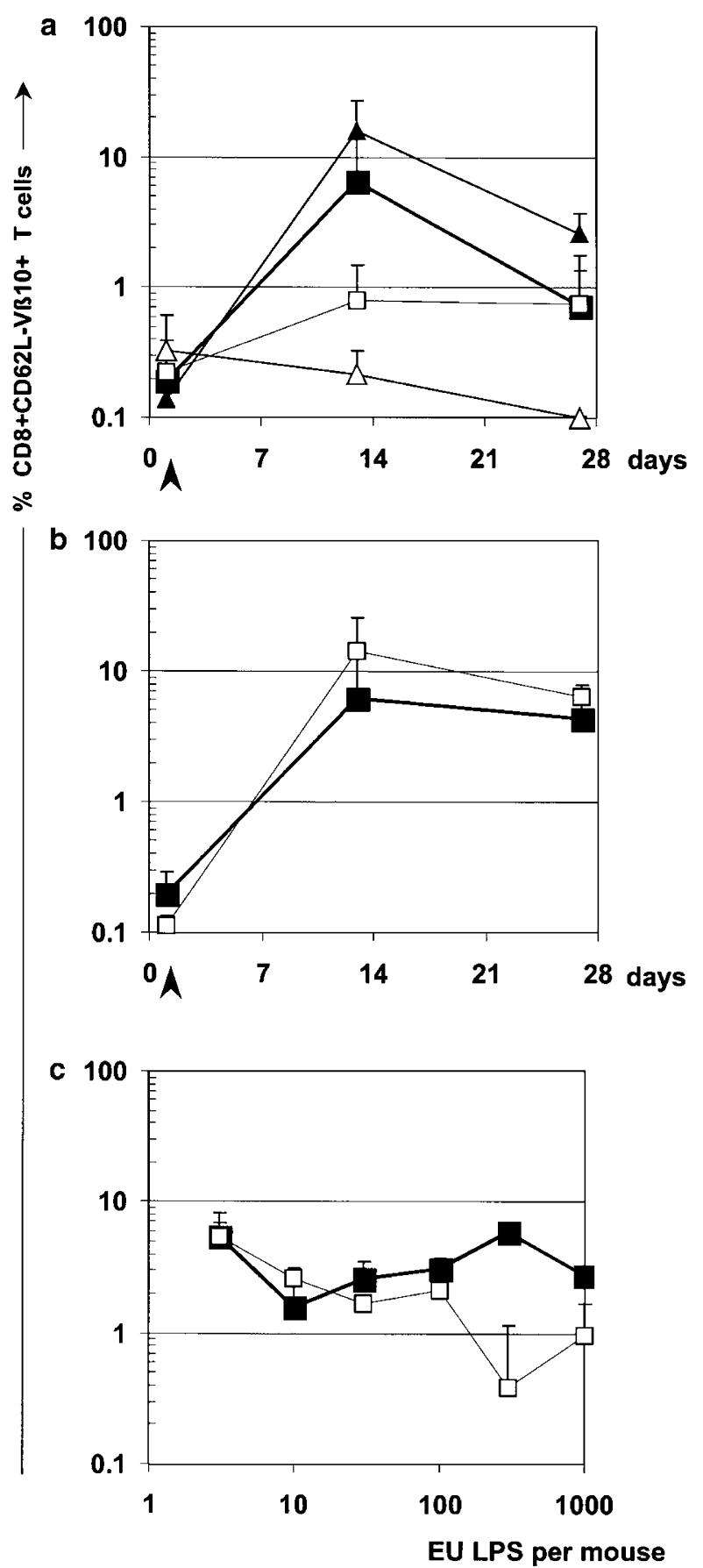

Figure 5 Cp G content or LPS do not significantly influence the outcome of in vivo EP. (a) Mice were electroporated with the plasmids $p V A X . C w 3(\mathbf{\Delta})$,

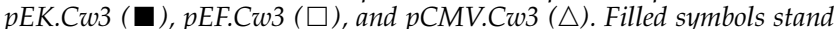
for plasmids with kanamycin and open symbols for ampicillin resistance; triangles stand for CMV promoter and squares for $h E F \alpha$ promoter. Groups of four mice received a single vaccination (arrowhead) with $50 \mu \mathrm{g}$ plasmid plus EP on day 1 and were repeatedly tested by FACS staining. Average+s.d. of the groups shown. (b) Each mouse was electrovaccinated (arrowhead) with $50 \mu \mathrm{g}$ pVAX.Cw3 plasmid plus $10 \mu \mathrm{g}$ of synthetic oligonucleotide with either a $\mathrm{C} p \mathrm{G}$ containing sequence (ם) or control sequence $(\square)$. (c) Mice were electroporated with mixtures of titrated amounts of E. coli LPS plus $50 \mu \mathrm{g}(\mathbf{\square})$ or $10 \mu \mathrm{g}(\square)$ of pVAX.Cw3 plasmids. CD8+ T-cell responses were read out on day 13; and average+s.d. of the groups are shown.

effects of high concentrations of LPS on immunization efficiency became apparent. Consistent with this, Boyle et al have shown that LPS has no effects on cellular and

humoral immune responses especially following i.m. DNA vaccination..$^{30}$ Weeratna et al showed that coadministered CpG oligos can interfere with in vivo transfection. ${ }^{31}$ In contrast, other inefficient DNA immunization protocols have been shown to be strongly enhanced at least by admixed CpG oligos. ${ }^{20}$ Besides these published reports, it is clear that the effects of these adjuvants for DNA vaccination were hardly visible in our highly efficient response with its very pronounced expansion of specific CD8+ T cells. Thus, apparently DNA vaccination can be optimized to a point where naked plasmid DNA alone is sufficient for immunization.

While the mechanisms of DNA vaccination itself are becoming evident, most of the reasons for the high efficiency of the Cw3 response still remain elusive. Cw3 as antigen is clearly an exceptional one, and new data indicate that besides the dominant RYLK epitope and its immediate surroundings, there is contribution from other distal structural elements of the Cw3 molecule. ${ }^{32}$ Additionally, we have published the first encouraging EP data in different mouse strains with different epitopes, derived from the TRP family of melanoma antigens. ${ }^{33}$ Thus, we believe that EP will be broadly applicable.

In vivo EP of Cw3-encoding plasmids is the first example of a purely molecular vaccine that induces highdegree CD8+ T-cell expansion with a long-lived memory upon a single administration. While a number of aspects are still unclear and have to be addressed, the parameters of EP are closely approaching an ideal vaccine. This should encourage investments in optimizing EP protocols for future human use.

\section{Methods \\ Mice}

Six-to-eight-week-old Female DBA/2 and B6D2F1 hybrid mice were purchased from Harlan, Germany and held under specified pathogen-free conditions. Mice were acclimated 1 week before the start of the study. All animal work was performed in full compliance to institutional and legal guidelines.

\section{Cells}

The murine colon carcinoma cell line C-26 was a kind gift of Mario Colombo (INT Milano). The murine mastocytoma cell line P815 and the green monkey transformed fibroblast line COS7 were from ATCC (Manassas, VA, USA). The P815-Cw3 \#444/C931 line that carries the HLA-Cw3 genomic fragment was a kind gift of J-C Cerottini (LICR Lausanne, Switzerland). Cells that express the Cw3 epitope fused to EGFP were developed by stable transfection of plasmid 123 (see 'Plasmids and immunizations') into the P815 mastocytoma cell line. Transfectants were selected in $1.5 \mathrm{mg} / \mathrm{ml} \mathrm{G} 418$, sorted for high EGFP expression, and cloned by limiting dilution. A clone with stable high EGFP expression and favorable growth properties was named P815\#123, and used for tumor challenge experiments. CTLs were generated by in vitro restimulation of CD8+ spleen cells using irradiated P815-Cw3 cells in the presence of $30 \mathrm{U} /$ $\mathrm{ml}$ recombinant IL-2. 


\section{Immunogens and immunizations}

Plasmid pVAX.Cw3 was obtained by inserting an RTPCR product of the full-length HLA-Cw*0303 coding sequence into the low-CpG-containing vaccination vector pVAX1 (Invitrogen, Carlsbad, CA, USA). A fragment encoding the human growth hormone was cloned analogously to yield pVAX.hGH. ${ }^{34}$ Vector pEF6 (ampicillin resistance) was also from Invitrogen; by swapping promoter and resistance-gene regions was pEK.Cw3 (EF $\alpha$ promoter, kanamycin resistance) and pCMV.Cw3 (CMV promoter, ampicillin resistance) generated. For plasmid 123, a pair of complementary synthetic oligonucleotides encoding for the presented epitope RYLKNGKETL were annealed and inserted in frame into the multiple cloning site of pEGFPC1 (B-D Clontech). Synthetic oligonucleotides were prepared according to Davis et al: ${ }^{20}$ oligo 1826 from the above publication was used as $\mathrm{CpG}$-containing positive control and oligo 1982 as non-CpG negative control. Peptides were synthesized by solid-phase technology and purified by reversed-phase HPLC. Baculoviruses were constructed similar to those described by Condreay et al with vBACw3 containing the EGFP-RYLK epitope fusion sequence of plasmid 123 and vBACMV3 containing the full-length insert of plasmid pVAX.Cw3. ${ }^{14}$ Live baculoviruses were isolated on sucrose gradients and applied at 1E8 MOI per animal.

Plasmids were either purified on $\mathrm{CsCl}$ gradients or custom manufactured by ELIM Pharmaceuticals and contained less than $5 \mathrm{EU} / \mathrm{mg}$ endotoxin. Required amounts of plasmids were diluted into $100 \mu \mathrm{l} 20 \mathrm{mM}$ HEPES pH 7.4 buffer and $50 \mu \mathrm{l}$ were applied to both quadriceps femoris muscle of DBA/2 mice. Immediately six pulses at $60 \mathrm{~V}$ were applied using the BTX T820 device (Genetronics, San Diego CA, USA). All animal work was performed following institutional and legal guidelines. Optimization of in vivo EP settings with the aim to maximize transgene expression was started with the setup of Genetronics. ${ }^{9}$ Formulation, handling, and electroporator settings were further optimized with plasmids carrying luciferase and $\beta \mathrm{Gal}$ reporter genes for convenient ex vivo detection of transgene expression. Plasmids coding for human growth hormone (hGH) were also used as cosignal, easily detectable by ELISA in the serum.

\section{FACS analysis}

Blood $(150-200 \mu \mathrm{l})$ was sampled from the retro-orbital sinus using heparinized capillaries and tubes. Samples from each animal were at all steps processed and analyzed individually, without pooling. Lymphocytes were isolated by gradient density centrifugation (Lymphoprep; Nycomed AS). Mononuclear cell yield after micro-Lymphoprep was $2-5 \times 10^{5}$ per bleed. Normal rabbit immunoglobulin and anti-CD16/32 mAb were added to block unspecific sites first, followed by a titrated mixture of anti-CD62L-FITC, anti-CD8-PE, and anti-Vß10-biotin (all antibodies from B-D Pharmingen). APC- or CyChrome-conjugated streptavidin (both from Pharmingen) was used as second step reagents. CeIIs were analyzed on a FACSort or B-D LSR cytometer, and more than 300000 events gated on PBL were collected. Data were analyzed using WinMDI (J Trotter, Scripps Institute) and percent specific CD8+ cells calculated in two steps. ${ }^{35}$ First live, CD8+ cells were electronically gated. Then on a CD62L ( $x$-axis) versus V $\beta 10$ ( $y$-axis) dot plot displaying these gated cell only, four quadrants were set that defined high- and low-expressing cells. In order to obtain precise, unbiased values, results were corrected for the low-level spontaneous loss of CD62L. Background (LOD) measured on $>500$ occasions on naive, untreated or other control animals was $0.3 \pm 0.1 \%$.

\section{IFN- $\gamma$-release}

C-26 murine colon carcinoma cells were grown overnight in 96-well plates at 15000 cells per well and transiently transfected with $500 \mathrm{ng}$ DNA per well using the DEAEdextran/chloroquin method in triplicate cultures. After $3.5 \mathrm{~h}$ incubation, the transfection mixture was removed from the cells and fresh media were added. The day after transfection, 15000 HLA-Cw3-specific CTLs were added per well. After 4 days of cocultivation, supernatants were analyzed for their IFN- $\gamma$ content by ELISA. For quantification of IFN- $\gamma$ in cell culture supernatants, the OPTEIA Mouse IFN- $\gamma$ antibody set (Pharmingen) was used.

\section{Acknowledgements}

We thank J-C Cerottini, A Himmler, and W Günzburg for reagents, E Wagner and J Maryanski for their discussions at the initial phase of the project, and T Decker and GR Adolf for their suggestions and support.

\section{References}

1 Murali-Krishna K et al. Counting antigen-specific CD8 T cells: a reevaluation of bystander activation during viral infection. Immunity 1998; 8: 177-187.

2 Hou $\mathrm{S}$ et al. Virus-specific CD8+ T-cell memory determined by clonal burst size. Nature 1994; 369: 652-654.

3 MacDonald HR et al. Oligoclonal expansion of major histocompatibility complex class I-restricted cytolytic $\mathrm{T}$ lymphocytes during a primary immune response in vivo: direct monitoring by flow cytometry and polymerase chain reaction. J Exp Med 1993; 177: 1487-1492.

4 Casanova JL et al. H-2-restricted cytolytic T lymphocytes specific for HLA display T cell receptors of limited diversity. J Exp Med 1992; 176: 439-447.

5 Maryanski JL et al. Single-cell PCR analysis of TCR repertoires selected by antigen in vivo: a high magnitude CD8 response is comprised of very few clones. Immunity 1996; 4: 47-55.

6 Bradley LM et al. Characterization of antigen-specific CD4+ effector $\mathrm{T}$ cells in vivo: immunization results in a transient population of MEL-14-, CD45RB-helper cells that secretes interleukin 2 (IL-2), IL-3, IL-4, and interferon gamma. J Exp Med 1991; 174: 547-559.

7 Walker PR et al. Distinct phenotypes of antigen-selected CD8 T cells emerge at different stages of an in vivo immune response. J Immunol 1995; 155: 3443-3452.

8 Rizzuto G et al. Efficient and regulated erythropoietin production by naked DNA injection and muscle electroporation. Proc Natl Acad Sci USA 1999; 96: 6417-6422.

9 Widera $\mathrm{G}$ et al. Increased DNA vaccine delivery and immunogenicity by electroporation in vivo. I Immunol 2000; 164: 4635-4640.

10 Zucchelli S et al. Enhancing B- and T-cell immune response to a hepatitis c virus E2 DNA vaccine by intramuscular electrical gene transfer. J Virol 2000; 74: 11598-11607. 
11 Buschle $\mathrm{M}$ et al. Transloading of tumor antigen-derived peptides into antigen-presenting cells. Proc Natl Acad Sci USA 1997; 94: 3256-3261.

12 Schmidt W et al. Transloading of tumor cells with foreign MHC I peptide ligand: a novel general strategy for the generation of potent cancer vaccines. Proc Natl Acad Sci USA 1996; 93: 9759-9763.

13 Deres $\mathrm{K}$ et al. In vivo priming of virus-specific cytotoxic $\mathrm{T}$ lymphocytes with synthetic lipopeptide vaccine. Nature 1989; 342: $561-564$

14 Condreay JP et al. Transient and stable gene expression in mammalian cells transduced with a recombinant baculovirus vector. Proc Natl Acad Sci USA 1999; 96: 127-132.

15 Hofmann $C$ et al. Efficient gene transfer into human hepatocytes by baculovirus vectors. Proc Natl Acad Sci USA 1995; 92: 10099-10103.

16 Busch DH et al. Coordinate regulation of complex $\mathrm{T}$ cell populations responding to bacterial infection. Immunity 1998; 8: 353-362.

17 Hemmi $\mathrm{H}$ et al. A Toll-like receptor recognizes bacterial DNA. Nature 2000; 408: 740-745.

18 Sato $\mathrm{Y}$ et al. Immunostimulatory DNA sequences necessary for effective intradermal gene immunization. Science 1996; 273: 352-354.

19 Mizushima S, Nagata S. pEF-BOS, a powerful mammalian expression vector. Nucleic Acids Res 1990; 18: 5322-5322.

20 Davis HL et al. CpG DNA is a potent enhancer of specific immunity in mice immunized with recombinant hepatitis B surface antigen. J Immunol 1998; 160: 870-876.

21 Cotten $\mathrm{M}$ et al. Lipopolysaccharide is a frequent contaminant of plasmid DNA preparations and can be toxic to primary human cells in the presence of adenovirus. Gene Ther 1995; 1: 239-246.

22 Aliprantis AO et al. Cell activation and apoptosis by bacterial lipoproteins through toll-like receptor-2. Science 1999; 285: 736-739.

23 Tapping RI et al. Toll-like receptor 4, but not toll-like receptor 2, is a signaling receptor for Escherichia and Salmonella lipopolysaccharides. J Immunol 2000; 165: 5780-5787.

24 BenMohamed L et al. Lipopeptide immunization without adjuvant induces potent and long-lasting $\mathrm{B}, \mathrm{T}$ helper, and cytotoxic $\mathrm{T}$ lymphocyte responses against a malaria liver stage antigen in mice and chimpanzees. Eur J Immunol 1997; 27: $1242-1253$

25 Schneider $\mathrm{J}$ et al. Enhanced immunogenicity for CD8+ T cell induction and complete protective efficacy of malaria DNA vaccination by boosting with modified vaccinia virus Ankara. Nat Med 1998; 4: 397-402.

26 Barouch DH et al. Control of viremia and prevention of clinical AIDS in rhesus monkeys by cytokine-augmented DNA vaccination. Science 2000; 290: 486-492.

27 Mir LM et al. Long-term, high level in vivo gene expression after electric pulse-mediated gene transfer into skeletal muscle. C R Acad Sci III 1998; 321: 893-899.

28 Aihara H, Miyazaki J. Gene transfer into muscle by electroporation in vivo. Nat Biotechnol 1998; 16: 867-870.

29 Akbari $\mathrm{O}$ et al. DNA vaccination: transfection and activation of dendritic cells as key events for immunity. J Exp Med 1999; 189: 169-178.

30 Boyle JS et al. Inhibitory effect of lipopolysaccharide on immune response after DNA immunization is route dependent. DNA Cell Biol 1998; 17: 343-348.

31 Weeratna $\mathrm{R}$ et al. Reduction of antigen expression from DNA vaccines by coadministered oligodeoxynucleotides. Antisense Nucl Acid Drug Dev 1998; 8: 351-356.

32 Paster $\mathrm{W}$ et al. Structural elements of a protein antigen determine immunogenicity of the embedded MHC class I-restricted T cell epitope. J Immunol 2002; 169: 2937-2946.

33 Kalat $\mathrm{M}$ et al. In vivo plasmid electroporation induces tumor antigen-specific CD8+ T cell responses and delays tumor growth in a syngeneic mouse melanoma model. Cancer Res 2002; 62: 5489-5494.

34 Gunzburg WH et al. A mammary-specific promoter directs expression of growth hormone not only to the mammary gland, but also to Bergman glia cells in transgenic mice. Mol Endocrinol 1991; 5: 123-133.

35 Attuil V et al. Comparative $\mathrm{T}$ cell receptor repertoire selection by antigen after adoptive transfer: a glimpse at an antigen-specific preimmune repertoire. Proc Natl Acad Sci USA 2000; 97: 8473-8478. 